\title{
Frequency-domain equalizers with zero restoration for zero-padded block transmission with high SNR
}

\author{
Gert Cuypers ${ }^{*}$ and Marc Moonen
}

\begin{abstract}
This paper focuses on the equalization of block transmissions with zero pad (ZP). If the channel impulse response length does not exceed the ZP length, it is possible to construct zero-forcing equalizers (ZFEs). Improved performance may be achieved using a minimum mean squared error (MMSE) equalizer. However, these equalizers are computationally intensive when a time-domain implementation is used. While the frequency-domain implementation of a ZFE has a lower complexity, it is prone to-potentially infinite-noise enhancement in the presence of spectral zeros. The MMSE equalizer in the frequency domain performs better by limiting the noise enhancement but still loses all information stored at the spectral zeros. This paper presents a method to exploit the redundancy of the padding to recover this lost information, leading to two new frequency-domain equalizers, a ZFE and an MMSE-like equalizer. These two equalizers are evaluated through simulations. They have a performance close to the time-domain equalizers, while maintaining the low complexity of the original frequency-domain equalizers. The equalizers are especially useful for scenarios with a high signal-to-noise ratio (SNR), where the performance is not limited by the presence of noise, but by the absence of the information stored in the spectral zeros. In practice, this means an SNR $>30 \mathrm{~dB}$. With minor modifications, the equalizers can also be applied if the ZP is replaced by a unique word (UW).
\end{abstract}

Keywords: Equalization, Spectral zero, ZFE, MMSE, OFDM, Single-carrier, Block transmission, Zero padding

\section{Introduction}

The idea of using a discrete Fourier transform (DFT) to (de)modulate data on carriers-referred to as tones or subcarriers-goes back a long time [1]. The transmission is organized in blocks and operates as follows: each tone is assigned a complex data symbol corresponding to a constellation point. These complex data symbols for all tones are packed together in a vector which is converted to the time domain by means of an inverse DFT (IDFT). The resulting time-domain waveform can be seen as a summation of orthogonal signals-one for each tonehence the name orthogonal frequency division multiplexing (OFDM). This waveform is now transmitted over the channel. The received samples are stored in a vector which is converted back to the frequency domain using a DFT. Unfortunately, the channel causes inter-symbol interference and destroys the orthogonality between the

*Correspondence: gert.cuypers@esat.kuleuven.be

Esat, KU Leuven, Kasteelpark Arenberg 10, B-3001 Leuven, Belgium tones, complicating the equalization. Both problems can be solved by introducing a cyclic prefix (CP): a block of samples at the end of the time-domain waveform is copied and added in front of the waveform prior to transmission [2]. At the receiver side, the samples pertaining to the $\mathrm{CP}$ are discarded. The resulting effect is that the linear channel convolution now appears to be circular. Accordingly, the corresponding channel matrix in the frequency domain becomes a diagonal one and the received symbols on each tone only depend on the transmitted symbols on that tone. Equalization can now easily be done by multiplying with the inverse of the diagonal channel matrix. A good overview is given in [3-5].

Later on, it was realized that this equalization technique is not limited to OFDM but also applicable to single-carrier block transmissions (SC), see [6], who observes that this idea was already suggested two decades earlier [7]. 
Since one can be converted into the other by merely adding an orthogonal precoding, OFDM and SC have many similarities and mathematical techniques for one of them can usually be adapted for use with the other. The differences mostly concern practical implementation issues: because SC systems generally have a lower peak-to-average power ratio (PAPR) and a better robustness against non-linearities or carrier offset $[6,8,9]$, their demands on the analog hardware are lower than those of OFDM. Adaptive OFDM, on the other hand, has the advantage of allowing bit loading and power loading of each sub-carrier according to its quality [9].

Furthermore, adding a $\mathrm{CP}$ is not the only way to convert the linear channel convolution into a circular one. This can also be obtained by adding a zero pad (ZP) or unique word ( $\mathrm{UW})$ to the waveforms in the time domain. Specifically, a CP system relies on the overlap-save (OLS) technique, while a ZP system uses overlap-add (OLA) to obtain a circular convolution. Although $\mathrm{CP}$ and ZP systems appear to be interchangeable, there are important differences. More specifically, if the channel frequency response exhibits spectral zeros on or zeros near the unit circle, zero-forcing equalization may be impossible for a CP system or may lead to severe noise enhancement $[10,11]$. A CP-OFDM system can take this into account by discarding the affected sub-carriers if the channel is known at the transmitter or by using forward error correction (FEC) codes if it is not $[8,11]$. The signal projection scheme from [12] offers a fundamental solution but is prohibitive in complexity, while the precoding technique from [13] requires the channel to remain constant over a period of multiple blocks. For a CP-SC system with a zero-forcing equalizer (ZFE), the presence of spectral zeros in the channel response is even worse, as the enhanced noise spreads out over the entire time-domain block. The classical solution is to limit the noise enhancement by using a minimum mean square error (MMSE) equalizer instead [14]. The information stored in the subcarriers corresponding to the zeros, however, will still be lost.

For a ZP system, on the other hand, zero-forcing equalization is always possible $[11,15]$. Unfortunately, using the OLA technique to implement the ZFE in the frequency domain introduces the same vulnerability to spectral zeros in the channel response as in CP systems. This results in an ill-conditioned problem and again the need to revert to an MMSE equalizer. Again, the information stored in the sub-carriers corresponding to the zeros is still lost. A decision feedback equalizer (DFE) on sample level might solve this problem. However, due to the processing in the time domain, its implementation will be much less efficient than the frequency-domain techniques mentioned before.
This paper presents two new frequency-domain equalizers for ZP transmission which can recover the information lost in the spectral zeros, while maintaining a complexity, comparable to frequency-domain solutions. They are applicable to OFDM as well as SC transmissions. The equalizers are analysed through simulation for several realistic scenarios, including situations where the performance is not limited by the signal-to-noise ratio (SNR), but by the missing information of the spectral zero. With minor modifications, they can also be used for systems using a UW.

The paper is organized is as follows: in Section 2, the system model and existing equalization techniques are presented. In Section 3, the new frequency-domain equalizers are introduced. In Section 4, simulations results are presented. Finally, in Section 5, conclusions are summarized.

\section{System model and equalizers}

The following conventions are followed: bold lowercase letters denote vectors and bold uppercase letters denote matrices. Normal uppercase letters denote constants. (.) $)^{T},(.)^{\mathcal{H}},(.)^{\dagger}$ and $E\{$.$\} denote transpose, Hermi-$ tian, pseudo-inverse and expected value, respectively, and $\mathbf{A}\left(:,\left[l_{1} \ldots l_{\mathrm{k}}\right]\right)$ selects columns $l_{1} \ldots l_{\mathrm{k}}$ of $\mathbf{A}$. The DFT, IDFT, zero and identity matrix of size $K$ are represented by $\mathcal{F}_{\mathrm{K}}, \mathcal{I}_{\mathrm{K}}, \mathbf{0}_{\mathrm{K}}$ and $\mathrm{I}_{\mathrm{K}}$, respectively. Occasionally, for clarity, the dimensions of a matrix are indicated as $[.]_{\mathrm{K} \times \mathrm{L}}$.

Now, consider a finite impulse response (FIR) transmission channel $\mathbf{h}=\left[\begin{array}{llll}h_{0} & h_{1} & \ldots & h_{\mathrm{L}}\end{array}\right]^{T}$ of order $L$, which is known by both transmitter and receiver and which can be considered quasi-static over one block period. The transmitted block $\mathbf{x}=\left[x_{1} x_{2} \ldots x_{\mathrm{N}}\right]^{T}$ is followed by a ZP of length $P$, with $P \geq L$ to avoid inter-block interference (IBI). Without loss of generality, it is assumed that the variance of $\mathbf{x}$ equals unity, i.e. $E\left\{\mathbf{x x}^{\mathcal{H}}\right\}=\mathbf{I}_{\mathrm{N}}$ and that $P=L$. The received signal $\mathbf{y}$ is given as

$$
\mathbf{y}=\underbrace{\left[\begin{array}{ccc}
h_{0} & & \\
\vdots & & \\
& \ddots & \\
h_{\mathrm{L}} & & \\
& \ddots & h_{0} \\
& & \vdots \\
& & \\
& & h_{\mathrm{L}}
\end{array}\right]}_{\mathbf{H}_{(\mathrm{N}+\mathrm{L}) \times \mathrm{N}}} \mathbf{x}+\mathbf{n},
$$

with $\mathbf{H}$ the linear convolution matrix of $\mathbf{h}$ and $\mathbf{n}$ additive noise. Without loss of generality, it is assumed that the noise is zero-mean and white with variance $\sigma_{\mathrm{n}}^{2}$, i.e. $E\left\{\mathbf{n n}^{\mathcal{H}}\right\}=\sigma_{\mathrm{n}}^{2} \mathbf{I}_{\mathrm{N}+\mathrm{L}}$. 


\subsection{Time domain equalization}

An equalizer $\mathbf{W}$ is a matrix for which $\hat{\mathbf{x}}=\mathbf{W} \mathbf{y}$ is an estimate of $\mathbf{x}$. Moreover, any equalizer $\mathbf{W}_{\mathrm{ZFE}}$ that satisfies the following constraint

$$
\mathbf{W}_{\mathrm{ZFE}} \mathbf{H}_{(\mathrm{N}+\mathrm{L}) \times \mathrm{N}}=\mathbf{I}_{\mathrm{N}}
$$

is referred to as a zero forcing equalizer (ZFE). In the absence of noise, such ZFE obviously leads to a perfect reconstruction of the transmitted block. Because $\mathbf{H}$ is a tall Toeplitz matrix, an infinite number of equalizers exist that satisfy Eq. (2). Since $\hat{\mathbf{x}}=\mathbf{x}+\mathbf{W}_{\text {ZFE }} \mathbf{n}$, ZFEs only differ in the way they treat the noise. One particular ZFE is obtained as the pseudo-inverse of $\mathbf{H}$, which then provides the minimum-norm solution of Eq. (2). It is given by ${ }^{1}$

$$
\mathbf{W}_{\text {ZFE-TD }}=\mathbf{H}^{\dagger}=\left(\mathbf{H}^{\mathcal{H}} \mathbf{H}\right)^{-1} \mathbf{H}^{\mathcal{H}}
$$

Here, $\mathbf{H}^{\mathcal{H}} \mathbf{H}$ is a full-rank $N \times N$ matrix and hence always invertible. This is referred to as the ZFE-TD (time-domain ZFE) where 'time-domain' refers to the absence of a transformation to the frequency domain (see Section 2.2). It is the optimal ZFE because it has the smallest noise enhancement.

By dropping the perfect reconstruction constraint of Eq. (2), it is often possible to achieve improved performance. The MMSE equalizer also takes into account the noise statistics and performs an overall optimization by minimizing the expected value of the squared error, $E\left\{\left\|\mathbf{x}-\mathbf{W}_{\text {MMSE-TD } \mathbf{y}}\right\|^{2}\right\}$. It can be expressed as

$$
\mathbf{W}_{\text {MMSE-TD }}=\left(\mathbf{H}^{\mathcal{H}} \mathbf{H}+\sigma_{\mathrm{n}}^{2} \mathbf{I}_{\mathrm{N}}\right)^{-1} \mathbf{H}^{\mathcal{H}}
$$

The calculation of these equalizers has a complexity of $\mathcal{O}\left(N^{2}\right)$ flops (using structured matrix inversion formulae) while calculating $\hat{\mathbf{x}}=\mathbf{W y}$ requires no more than $\mathcal{O}\left(N^{2}\right)$ flops per block [16].

\subsection{Frequency-domain equalization based on matrix folding}

To exploit frequency-domain techniques, the linear channel convolution needs to be converted into a circular one. Accordingly, the matrix $\mathbf{H}$ needs to be transformed into a circular matrix. This can be achieved in two ways: either by folding the last $L$ rows back to the top or by extending it with $L$ more columns. The first method corresponds to an overlap-add operation: the last $L$ samples of $\mathbf{y}$ are added to the first $L$ samples and then only the first $N$ samples of the result are kept, resulting in

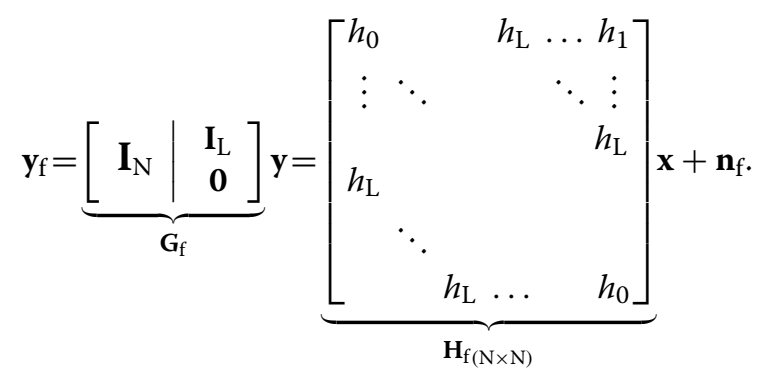

Note that the multiplication with the $(N \times(N+L))$ matrix $\mathbf{G}_{\mathrm{f}}$ reduces the number of matrix rows and hence the information in the system. The existence of a ZFE solution may therefore no longer be guaranteed. Indeed, $\mathbf{H}_{\mathrm{f}}$ is an $N \times N$ matrix which becomes rank deficient if the Z-transform of $\mathbf{h}$ has a zero on one of the $N$ equally spaced points $e^{\frac{2 \pi i}{N} k}$, with $k=0 \ldots N-1$, referred to as spectral zeros. If $\mathbf{H}_{\mathrm{f}}$ has rank $N$, the resulting unique ZFE can be written as

$$
\mathbf{W}_{\text {ZFE-TD-FOLD }}=\mathbf{H}_{\mathrm{f}}^{-1} \mathbf{G}_{\mathrm{f}} \text {. }
$$

To perform the equalization in the frequency domain, note that $\mathbf{H}_{\mathrm{f}}$ can be diagonalized to $\Lambda_{\mathrm{f}}$, such that Eq. (5) becomes

$$
\mathbf{y}_{\mathrm{f}}=\underbrace{\mathcal{I}_{\mathrm{N}} \Lambda_{\mathrm{f}} \mathcal{F}_{\mathrm{N}}}_{\mathbf{H}_{\mathrm{f}}} \mathbf{x}+\mathbf{n}_{\mathrm{f}} .
$$

The frequency-domain ZFE can now be written as

$$
\mathbf{W}_{\text {ZFE-FD-FOLD }}=\underbrace{\mathcal{I}_{\mathrm{N}} \Lambda_{\mathrm{f}}^{-1} \mathcal{F}_{\mathrm{N}}}_{\mathbf{H}_{\mathrm{f}}^{-1}} \mathbf{G}_{\mathrm{f}} .
$$

The calculation of the inversion of the diagonal matrix in this equation requires $\mathcal{O}(N)$ flops, whereas the calculation of $\hat{\mathbf{x}}$ requires only $\mathcal{O}(N \log (N))$ flops per block, if the (I)DFT operation is implemented using the (inverse) fast Fourier transform. Obviously, an important saving is to be made compared to the ZFE-TD, especially for large values of $N$. However, this speedup comes at a cost: if the channel has spectral zeros or zeros near the unit circle, the inversion of $\Lambda_{\mathrm{f}}$ may be impossible or lead to very large values and hence a significant noise enhancement.

This problem is well known, and several authors have proposed the MMSE approach to limit the detrimental influence of the noise [12, 17], see [18] for the OFDM case. The frequency-domain MMSE equalizer can be expressed as

$\mathbf{W}_{\text {MMSE-FD-FOLD }}=\mathcal{I}_{\mathrm{N}}\left(\Lambda_{\mathrm{f}}^{\mathcal{H}} \Lambda_{\mathrm{f}}+\sigma_{\mathrm{n}}^{2}\left(\frac{N+L}{N}\right) \mathbf{I}_{\mathrm{N}}\right)^{-1} \Lambda_{\mathrm{f}}^{\mathcal{H}} \mathcal{F}_{\mathrm{N}} \mathbf{G}_{\mathrm{f}}$ 
where the regularization term $\left(\frac{N+L}{N}\right)$ reflects the difference in noise shaping due to $\mathbf{G}_{\mathrm{f}}$.

\subsection{Frequency-domain equalization based on matrix extension}

It is also possible to add appropriate columns to $\mathbf{H}$ to extend it to a circular $(N+L) \times(N+L)$ matrix $\mathbf{H}_{\mathrm{e}}$. Equation (1) is then extended to explicitly involve the ZP in the calculations, i.e.

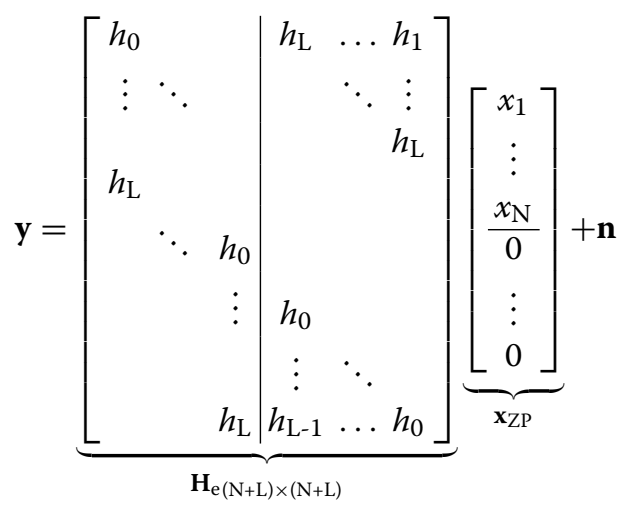

Note that $\mathbf{H}_{\mathrm{e}}$ is an $M \times M$ matrix, with $M=N+L$, of which the rank is minimally $N$. Now, $\mathbf{H}_{\mathrm{e}}$ can be diagonalized to $\Lambda_{\mathrm{e}}$, having diagonal elements $\lambda_{\mathrm{e}_{1}}, \lambda_{\mathrm{e}_{2}}, \ldots \lambda_{\mathrm{e}_{\mathrm{M}}}$, yielding

$$
\mathbf{y}=\underbrace{\mathcal{I}_{\mathrm{M}} \Lambda_{\mathrm{e}} \mathcal{F}_{\mathrm{M}}}_{\mathbf{H}_{\mathrm{e}}} \mathbf{x}_{\mathrm{ZP}}+\mathbf{n} .
$$

The frequency-domain ZFE can be written as

$$
\mathbf{W}_{\text {ZFE-FD-EXT }}=\left[\mathbf{I}_{\mathrm{N}} \mid \mathbf{O}\right] \mathcal{I}_{\mathrm{M}} \Lambda_{\mathrm{e}}^{-1} \mathcal{F}_{\mathrm{M}}
$$

The calculation of the inversion of the diagonal matrix in this equation requires $\mathcal{O}(M)$ flops, whereas the calculation of $\hat{\mathbf{x}}$ requires $\mathcal{O}(M \log (M))$ flops per block. Note that, in the absence of spectral zeros, there exists a clever method to express the optimal ZFE, $\mathbf{W}_{\text {ZFE-TD }}$ in terms of $\mathbf{W}_{\text {ZFE-FD-EXT }}$ and a correction term of low complexity [19].

In case of spectral zeros at one of the points $e^{\frac{2 \pi i}{N+L} k}$, with $k=0 \ldots M-1, \Lambda_{\mathrm{e}}$ may be a rank-deficient matrix. Again, this can be dealt with by using the MMSE equalizer,

$\mathbf{W}_{\text {MMSE-FD-EXT }}=\left[\mathbf{I}_{\mathrm{N}} \mid \mathbf{O}\right] \mathcal{I}_{\mathrm{M}}\left(\Lambda_{\mathrm{e}}^{\mathcal{H}} \Lambda_{\mathrm{e}}+\sigma_{\mathrm{n}}^{2} \mathbf{I}_{\mathrm{M}}\right)^{-1} \Lambda_{\mathrm{e}}^{\mathcal{H}} \mathcal{F}_{\mathrm{M}}$

It is remarkable that the assured possibility of zeroforcing equalization is lost in going from Eq. (1) to Eq. (12), because the number of equations is unchanged (contrary to Eq. (5), where information is truly discarded). The problem arises in Eq. (10) when the matrix $\mathbf{H}_{\mathrm{e}}$ is not of full rank. For the solution in the time domain, however, $\mathbf{H}_{\mathrm{e}}$ does not need to be of full rank because the last $L$ elements of $\mathbf{x}_{\mathrm{ZP}}$ are known to be zero. In the frequency domain, this additional information is unavailable. In the next section, this problem is solved by feeding back this known information and fixing the possible 'gaps' in $\Lambda_{\mathrm{e}}$, thereby restoring the information that was lost due to the spectral zeros.

\section{Improved frequency-domain equalization}

In Section 2.3, the ZP part of the equalized signal is discarded by the multiplication with $\left[\mathbf{I}_{\mathrm{N}} \mid \mathbf{O}\right]$ in Eqs. (12) and (13). The equalized ZP, however, contains information that can help to improve the equalization of the useful signal.

Before diving into the mathematics, the general idea will be illustrated by a graphical example. Consider a simple binary communication system with zero padding. The elements of $\mathbf{x}$ only take two values, as shown in Fig. 1 .

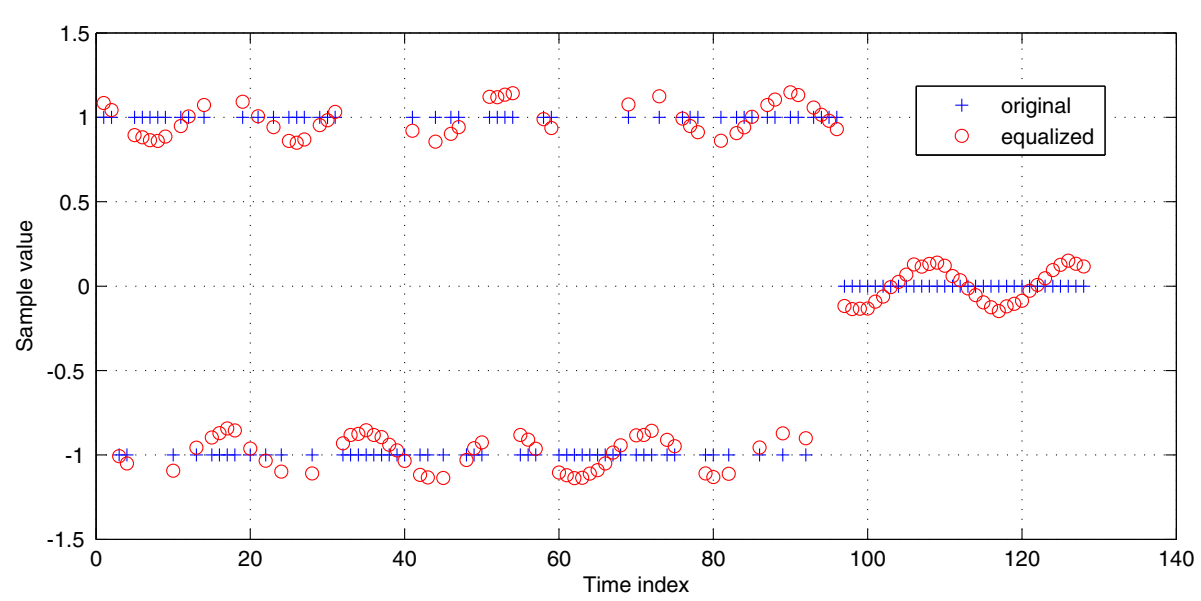

Fig. 1 The originally transmitted signal and the equalized received signal, assuming one sub-carrier was discarded. The phase and amplitude of the missing sub-carrier can be estimated by inspecting the zero pad 
Assume a channel that can be properly equalized at every sub-carrier except for one, where it exhibits a spectral zero. Any energy received at the sub-carrier exhibiting this spectral zero can therefore only be noise and the best any frequency-domain equalizer can do is to discard it. Note that this is exactly what the MMSE equalizer in Eq. (13) does. The resulting $\hat{\mathbf{x}}$, including the equalized $\mathrm{ZP}$, is also shown in Fig. 1. Comparing the transmitted signal and the equalized version, a sinusoidal disturbance is immediately apparent. This disturbance is caused by the absence of the contribution of the sub-carrier which exhibited a spectral zero. If this missing contribution would be added, the equalized useful signal points would be closer to the transmitted ones, and the equalized zero pad would be closer to zero. Exactly this last observation suggests a method to determine the contribution of the missing sub-carrier: its phase and amplitude should be chosen so as to minimize the zero pad. After determining these parameters, the contribution of this sub-carrier can be added. It is expected that this addition will also improve the quality of the useful signal. This idea will now be elaborated mathematically. To simplify the notation, it is assumed that $L=P$, which can easily be obtained by padding $\mathbf{h}$ with zeros.

\subsection{Frequency-domain ZFE with zero restoration}

If $\Lambda_{\mathrm{e}}$ is invertible and well-conditioned, the solutions from Eqs. (12) or (13) can be used. If $\Lambda_{\mathrm{e}}$ is rank-deficient or ill-conditioned, assume $V$ diagonal elements of $\Lambda_{\mathrm{e}}$ are non-zero while the remaining $K=M-V$ elements are zero or close to zero. Note that, because $\mathbf{H}_{\mathrm{e}}$ is at least of rank $N$, it follows that $K \leq P$. Now rearrange the diagonal elements of $\Lambda_{\mathrm{e}}$ such that the $K$ (closeto-) zero elements are collected at the end. Rearrange the rows of the DFT-matrix $\mathcal{F}_{M}$ in a likewise manner and call the result $\mathbf{F}$. The matrix $\mathbf{H}_{\mathrm{e}}$ can now be diagonalized as

$$
\mathbf{H}_{\mathrm{e}}=\underbrace{\left[\begin{array}{c|c}
\mathbf{F}_{\mathrm{A}}^{\mathcal{H}} & \mathbf{F}_{\mathrm{C}}^{\mathcal{H}} \\
\hline \mathbf{F}_{\mathrm{B}}^{\mathcal{H}} & \mathbf{F}_{\mathrm{D}}^{\mathcal{H}}
\end{array}\right]}_{\mathbf{F}^{\mathcal{H}}}\left[\begin{array}{c|c}
\Lambda_{\mathrm{nz}} & \mathbf{O} \\
\hline \mathbf{O} & \Lambda_{\epsilon}
\end{array}\right] \underbrace{\left[\begin{array}{c|c}
\mathbf{F}_{\mathrm{A}} & \mathbf{F}_{\mathrm{B}} \\
\hline \mathbf{F}_{\mathrm{C}} & \mathbf{F}_{\mathrm{D}}
\end{array}\right]}_{\mathbf{F}},
$$

in which $\Lambda_{\mathrm{nz}}$ holds the non-zero diagonal elements of $\Lambda_{\mathrm{e}}, \Lambda_{\epsilon}$ holds the (close-to-) zero elements and $\mathbf{F}_{\mathrm{A}(\mathrm{V} \times \mathrm{N})}$, and $\mathbf{F}_{\mathrm{B}(\mathrm{V} \times \mathrm{L})}, \mathbf{F}_{\mathrm{C}(\mathrm{K} \times \mathrm{N})}$ and $\mathbf{F}_{\mathrm{D}(\mathrm{K} \times \mathrm{L})}$ are submatrices of $\mathbf{F}$. Because $\mathbf{F}^{\mathcal{H}} \mathbf{F}=\mathbf{I}$, specific relations hold between these submatrices, e.g. $\mathbf{F}_{\mathrm{B}}^{\mathcal{H}} \mathbf{F}_{\mathrm{A}}=-\mathbf{F}_{\mathrm{D}}^{\mathcal{H}} \mathbf{F}_{\mathrm{C}}$ and $\mathbf{F}_{\mathrm{A}}^{\mathcal{H}} \mathbf{F}_{\mathrm{A}}+\mathbf{F}_{\mathrm{C}}^{\mathcal{H}} \mathbf{F}_{\mathrm{C}}=\mathbf{I}$.

Inverting $\Lambda_{\epsilon}$-if at all possible-would lead to a significant noise enhancement. However, by disregarding $\Lambda_{\epsilon}$ and inverting only $\Lambda_{\mathrm{nz}}$, a first approximation of $\mathbf{x}_{\mathrm{ZP}}$ can be calculated,

$$
\begin{aligned}
\hat{\mathbf{x}}_{\mathrm{ZP}_{\text {temp }}} & =\mathbf{F}^{\mathcal{H}}\left[\begin{array}{c|c}
\Lambda_{\mathrm{nz}}^{-1} & \mathbf{O} \\
\hline \mathbf{O} & \mathbf{O}
\end{array}\right] \mathbf{F y} \\
& =\left[\begin{array}{c}
\mathbf{F}_{\mathrm{A}}^{\mathcal{H}} \\
\hline \mathbf{F}_{\mathrm{B}}^{\mathcal{H}}
\end{array}\right] \Lambda_{\mathrm{nz}}^{-1}\left[\mathbf{F}_{\mathrm{A}} \mid \mathbf{F}_{\mathrm{B}}\right] \mathbf{y},
\end{aligned}
$$

Note that for high SNR values, this converges to the MMSE-solution of Eq. (13).

Because $\mathbf{F}^{\mathcal{H}}$ can be derived from $\mathcal{I}$ by a simple permutation of the rows and columns, it is also an orthogonal matrix and, as such, its columns form a base onto which the eventual equalizer output $\hat{\mathbf{x}}$ can be decomposed. Most of the coefficients of this decomposition can be derived from Eq. (16); more specifically, these are the coefficients related to the first $V$ columns of $\mathbf{F}^{\mathcal{H}}$ and the result is $\hat{\mathbf{x}}_{\mathrm{ZP}}$ temp . The coefficients related to the last $K$ columns of $\mathbf{F}^{\mathcal{H}}$ are unknown and set to zero in (16). However, including these columns could potentially lead to a better approximation of $\mathbf{x}$. Therefore, a correction term is added to $\hat{\mathbf{x}}_{\mathrm{ZP}}$ temp , which lies in the column space of the last $K$ columns of $\mathbf{F}^{\mathcal{H}}$, and which can be written as

$$
\hat{\mathbf{x}}_{\mathrm{ZP}_{\text {corr }}}=\left[\frac{\mathbf{F}_{\mathrm{C}}^{\mathcal{H}}}{\mathbf{F}_{\mathrm{D}}^{\mathcal{H}}}\right] \mathbf{q},
$$

where $\mathbf{q}$ holds the unknown coefficients related to the last $V$ columns of $\mathbf{F}^{\mathcal{H}}$. The correction term can be determined by taking into account that the last $P$ elements of $\hat{\mathbf{x}}_{\text {ZP }}$ should be zero. This leads to a system of $P$ equations in $K$ unknowns, which can be solved exactly if no noise is present. In practice, the correction term can be calculated from the following least-squares (LS) system:

$$
\mathbf{F}_{\mathrm{D}}^{\mathcal{H}} \mathbf{q}+\left[\mathbf{O} \mid \mathbf{I}_{\mathrm{L}}\right] \hat{\mathbf{x}}_{\mathrm{ZP}} \stackrel{L S}{\stackrel{L S}{=}} \mathbf{O}_{\mathrm{L} \times 1}
$$

the solution of which is given by

$$
\mathbf{q}=-\left(\mathbf{F}_{\mathrm{D}} \mathbf{F}_{\mathrm{D}}^{\mathcal{H}}\right)^{-1} \mathbf{F}_{\mathrm{D}} \mathbf{F}_{\mathrm{B}}^{\mathcal{H}} \Lambda_{\mathrm{nz}}^{-1}\left[\mathbf{F}_{\mathrm{A}} \mid \mathbf{F}_{\mathrm{B}}\right] \mathbf{y} .
$$

The overall expression for $\hat{\mathbf{x}}$ therefore becomes

$$
\begin{aligned}
\hat{\mathbf{x}} & =\left[\mathbf{I}_{N} \mid \mathbf{O}\right]\left(\hat{\mathbf{x}}_{\mathrm{ZP} \text { temp }}+\hat{\mathbf{x}}_{\mathrm{ZP} \text { corr }}\right) \\
& =\underbrace{\left(\mathbf{F}_{\mathrm{A}}^{\mathcal{H}}-\mathbf{F}_{\mathrm{C}}^{\mathcal{H}}\left(\mathbf{F}_{\mathrm{D}} \mathbf{F}_{\mathrm{D}}^{\mathcal{H}}\right)^{-1} \mathbf{F}_{\mathrm{D}} \mathbf{F}_{\mathrm{B}}^{\mathcal{H}}\right) \Lambda_{\mathrm{nz}}^{-1}\left[\mathbf{F}_{\mathrm{A}} \mid \mathbf{F}_{\mathrm{B}}\right] \mathbf{y}}_{\mathbf{W}_{\mathrm{ZFE}-\mathrm{ZR}}}
\end{aligned}
$$

This equation defines the ZFE-ZR, where ZR refers to zero restoration. It can be rewritten as

$$
\mathbf{W}_{\mathrm{ZFE}-\mathrm{ZR}}=\left[\begin{array}{ll}
\mathrm{F}_{\mathrm{A}}^{\mathcal{H}} & \mathrm{F}_{\mathrm{C}}^{\mathcal{H}}
\end{array}\right]\left[\begin{array}{c|c}
\mathbf{I} & \mathbf{O} \\
\hline \mathbf{Q} & \mathbf{O}
\end{array}\right]\left[\begin{array}{c|c}
\Lambda_{\mathrm{nZ}}^{-1} & \mathbf{O} \\
\hline \mathbf{O} & \mathbf{O}
\end{array}\right] \mathbf{F},
$$

with $\mathbf{Q}=-\left(\mathbf{F}_{\mathrm{D}} \mathbf{F}_{\mathrm{D}}^{\mathcal{H}}\right)^{-1} \mathbf{F}_{\mathrm{D}} \mathbf{F}_{\mathrm{B}}^{\mathcal{H}}=-\left(\mathrm{F}_{\mathrm{D}}^{\mathcal{H}}\right)^{\dagger} \mathbf{F}_{\mathrm{B}}^{\mathcal{H}}$. 
It can now be shown that $\mathbf{W}_{\text {ZFE-ZR }}$ satisfies the constraint of Eq. (2):

$$
\begin{aligned}
& \mathbf{W}_{\text {ZFE-ZR }} \mathbf{H} \\
& =\mathbf{W}_{\text {ZFE-ZR }} \mathbf{H}_{\mathrm{e}}\left[\begin{array}{c}
\mathbf{I} \\
\mathbf{O}
\end{array}\right] \\
& =\left[\mathrm{F}_{\mathrm{A}}-\mathrm{F}_{\mathrm{C}} \mathbf{Q} \mathbf{O}\right]\left[\begin{array}{c|c}
\Lambda_{\mathrm{nz}}^{-1} \Lambda_{\mathrm{nz}} & \mathbf{O} \\
\mathbf{O} & \mathbf{O} \Lambda_{\epsilon}
\end{array}\right]\left[\begin{array}{c}
\mathrm{F}_{\mathrm{A}} \\
\mathrm{F}_{\mathrm{C}}
\end{array}\right] \\
& =\mathbf{F}_{\mathrm{A}}^{\mathcal{H}} \mathbf{F}_{\mathrm{A}}+\mathbf{F}_{\mathrm{C}}^{\mathcal{H}} \underbrace{\left(\mathbf{F}_{\mathrm{D}} \mathbf{F}_{\mathrm{D}}^{\mathcal{H}}\right)^{-1} \mathbf{F}_{\mathrm{D}} \mathbf{F}_{\mathrm{D}}^{\mathcal{H}}}_{\mathbf{I}} \mathbf{F}_{\mathrm{C}} \\
& =\mathbf{I} .
\end{aligned}
$$

So the ZFE-ZR is indeed a ZFE, capable of recovering the lost information. Note that this derivation holds even if the diagonal elements of $\Lambda_{\epsilon}$ are not exactly equal to zero, which is more common in real-world situations.

Note that this procedure involves calculating the pseudo-inverse of $\mathrm{F}_{\mathrm{D}}^{\mathcal{H}}$, which is poorly conditioned if the corresponding columns of the IDFT matrix are close to each other. In other words, the restoration of the information lost in spectral (close-to-) zeros works best if these spectral (close-to-) zeros are not clustered.

The result of Eq. (21) has some similarity to another ZFE proposed in [20], which indeed targets the same problem of spectral zeros, be it in the context of a diversity analysis. In the current notation, this ZFE can be written as

$$
\mathbf{W}=\left[\mathrm{F}_{\mathrm{A}}^{-1} \mathbf{O}\right]\left[\begin{array}{c|c}
\Lambda_{\mathrm{nz}}^{-1} & \mathbf{O} \\
\hline \mathbf{O} & \mathbf{O}
\end{array}\right] \mathbf{F},
$$

which can easily be shown to satisfy Eq. (2) as well. Note that Eq. (23) assumes that $K=P$, because $\mathrm{F}_{\mathrm{A}}$ needs to be square. Under this assumption, the solutions of Eqs. (21) and (23) are mathematically identical, because of the uniqueness of $\mathbf{F}_{\mathrm{A}}^{-1}$. Regarding the computational complexity, however, they are very different. The evaluation of Eq. (23) requires the inversion of $\mathbf{F}_{\mathrm{A}}$. Taking into account the Vandermonde structure of this matrix, this requires $5 N^{2} / 2$ operations [20], which typically is much higher than the evaluation of Eq. (21), as will be shown later. Note that the same author also offered a similar solution, which was the first linear equalization scheme to achieve maximum multipath diversity over single-input single-output wireless links [21].

\subsection{Frequency-domain MMSE-like equalization with zero restoration}

As noted before, frequency-domain MMSE equalizers can avoid dramatic noise enhancement due to an illconditioned channel matrix. However, they too do not offer any solution for the loss of information associated with a (close-to-) zero on the diagonal of $\Lambda_{c}$. This can be solved using a structure similar to the ZFE-ZR presented in the previous section.
Using $\Lambda_{\mathrm{nz}}$ and $\Lambda_{\epsilon}$ from Eq. (14), the following diagonal matrices can be defined:

$$
\begin{aligned}
\Gamma_{\mathrm{nz}} & =\left(\Lambda_{\mathrm{nz}}^{\mathcal{H}} \Lambda_{\mathrm{nz}}+\sigma_{\mathrm{n}}^{2} \mathbf{I}_{\mathrm{V}}\right)^{-1} \Lambda_{\mathrm{nz}}^{\mathcal{H}} \\
\Gamma_{\epsilon} & =\left(\Lambda_{\epsilon}^{\mathcal{H}} \Lambda_{\epsilon}+\sigma_{\mathrm{n}}^{2} \mathbf{I}_{\mathrm{K}}\right)^{-1} \Lambda_{\epsilon}^{\mathcal{H}},
\end{aligned}
$$

such that the MMSE-FD-EXT in Eq. (13) can be rewritten as

$$
\mathbf{W}_{\text {MMSE-FD-EXT }}=\left[\begin{array}{ll}
\mathbf{F}_{\mathrm{A}}^{\mathcal{H}} & \mathbf{F}_{\mathrm{C}}^{\mathcal{H}}
\end{array}\right]\left[\begin{array}{c|c}
\Gamma_{\mathrm{nz}} & \mathbf{O} \\
\hline \mathbf{O} & \Gamma_{\epsilon}
\end{array}\right] \mathbf{F} .
$$

Following the same steps as in the previous section, an improved MMSE-like equalizer with zero restoration can be obtained as

$$
\mathbf{W}_{\text {MMSE-ZR }}=\left[\mathbf{F}_{\mathrm{A}}^{\mathcal{H}} \mathbf{F}_{\mathrm{C}}^{\mathcal{H}}\right]\left[\begin{array}{l|l}
\mathbf{I} & \mathbf{O} \\
\hline \mathbf{Q} & \mathbf{O}
\end{array}\right]\left[\begin{array}{c|c}
\Gamma_{\mathrm{nz}} & \mathbf{O} \\
\hline \mathbf{O} & \mathbf{O}
\end{array}\right] \mathbf{F},
$$

with exactly the same $\mathbf{Q}$ as in Eq. (21).

For a theoretical analysis of these two equalizers, we refer to the Appendix.

\subsection{Remarks}

Before proceeding to the simulation results, some additional remarks are in place:

\subsubsection{Threshold}

Practical channel knowledge is imperfect because it is based on estimates and hence spectral zeros will mostly be identified as spectral close-to-zeros. Therefore, a decision needs to be made as to which elements of $\Lambda_{e}$ should effectively be treated as zero, e.g. based on a threshold. This can be set at some percentage of the root mean square value of $\mathbf{h}$ or through some other method. In any case, it needs to be such that the number of zeros $K$ is not larger than $P$. A trade-off needs to be made: setting a low threshold and identifying too few spectral close-to-zeros can lead to noise enhancement. Setting a high threshold can lead to too many spectral close-to-zeros, all of whose received information is discarded.

In the case of reasonably high values of the SNR (e.g. $>30 \mathrm{~dB}$ ), elements of $\Lambda_{\mathrm{e}}$ with an absolute value below $\sigma_{\mathrm{n}}$ should be treated as zeros, because they have a major contribution to the MSE. In the case of non-white noise, the threshold should also take into account the SNR level at each sub-carrier.

\subsubsection{Condition}

Related to the previous point, it should be avoided to assign consecutive elements of $\Lambda_{\mathrm{e}}$ as zeros. This would lead to an LS system based on consecutive rows of a DFT submatrix, which can be poorly conditioned.

Both points should lead to some heuristic algorithm to determine the selection of the elements which will be treated as zeros, e.g. the approach used in the simulations 
section is to only retain the 'lowest' of a set of consecutive zeros.

Extensive simulations have shown that treating one element of $\Lambda_{\mathrm{e}}$ as a zero generally does no harm and has the potential to improve the performance significantly. Keeping in mind that spectral zeros are relatively rare, and multiple spectral zeros are even rarer, designating the lowest element of $\Lambda_{e}$ as a zero suffices for most 'difficult' channels. Even if this tone was carrying useful information, this can easily be recovered from the information in the other tones. On the other hand, in the extreme case, where $K=P$, Eq. (18) is no longer a LS system and will be modeling the noise as well as reconstruct the information stored in the spectral zeros. This should only be considered for relatively high values of the SNR.

\subsubsection{UW}

The zero-restoration equalizers can also be adapted to UW-based transmission, either by subtracting the contribution of the UW at the receiver and proceeding as in the ZP case or by replacing $\hat{\mathbf{x}}_{Z P}$ by $\hat{\mathbf{x}}_{U W}$ and modifying the LS system such that the last $P$ samples of $\hat{\mathbf{x}}_{\mathrm{UW}}$ equal the UW.

\subsubsection{Implementation}

Note that, while in Eq. (15) the last $K$ columns of $\mathbf{F}^{\mathcal{H}}$ are effectively multiplied by coefficients equal to zero, even non-zero coefficients may be used here, because these are then absorbed by $\mathbf{q}$ anyway. It is therefore also possible to choose coefficients equal to $\Lambda_{\epsilon}^{-1}$. This implies that the ZFE-ZR can be implemented as a post-processing of the ZFE-FD-EXT.

A practical implementation could therefore start with the evaluation of -without the premultiplication with $\left[\begin{array}{l|l}\mathbf{I}_{\mathrm{N}} & \mathbf{O}\end{array}\right]$ - as an alternative for $\hat{\mathbf{x}}_{\mathrm{ZP} \text { temp }}$. Next, the LS system from Eq. (18) is solved, which is typically very small. The calculation of $\mathbf{q}$ requires $\left(N+L+\frac{K}{3}\right) K^{2}$ flops ([22], p. 238) and the calculation of $\hat{\mathbf{x}}_{\mathrm{ZP}}$ corr requires another $(2 K-1) N$ flops to multiply $\mathbf{q}$ and $\mathbf{F}_{\mathrm{C}}^{\mathcal{H}}$. The overall computational complexity is thus equal to the complexity of Eq. (12) plus approximately $\mathcal{O}\left(\left(K^{2}+2 K\right) N\right)$ flops and is generally significantly smaller than the complexity of the time-domain equalizers of Eqs. (3) and (4). Likewise, the MMSE-ZR can be implemented as a post-processing of the MMSE-FD-EXT. Based on the measurement of the actual channel and the SNR, it can be decided to use the post-processing step or not.

Note that the ZFE-FD-FOLD and the MMSE-FD-FOLD cannot be used as a starting point of the ZR-algorithm because, contrary to $\mathbf{H}_{\mathrm{e}}, \mathbf{H}_{\mathrm{f}}$ is not guaranteed at least of rank $N$ and may not contain enough information to calculate $N$ unknowns. For an easy comparison, the complexity of all equalizers has been summarized in Table 1.
Table 1 Comparing the complexity for block size $N$, ZP-length $P$ and $K$ spectral zeros $(M=N+P)$

\begin{tabular}{lll}
\hline Equalizer & Calculating $\mathbf{w}$ & Evaluating $\hat{\mathbf{x}}$ \\
\hline $\mathbf{W}_{\text {ZFE-TD }}, \mathbf{W}_{\text {MMSE-TD }}$ & $\mathcal{O}\left(N^{2}\right)$ & $\mathcal{O}\left(N^{2}\right)$ \\
$\mathbf{W}_{\text {ZFE-FD-FOLD }}, \mathbf{W}_{\text {MMSE-FD-FOLD }}$ & $\mathcal{O}(N)$ & $\mathcal{O}(N \log (N))$ \\
$\mathbf{W}_{\text {ZFE-FD-EXT, }}, \mathbf{W}_{\text {MMSE-FD-EXT }}$ & $\mathcal{O}(M)$ & $\mathcal{O}(M \log (M))$ \\
$\mathbf{W}_{\text {ZFE-ZR }}, \mathbf{W}_{\text {MMSE-ZR }}$ & $\mathcal{O}(M)$ & $\mathcal{O}(M \log (M))$ \\
& $+\left(M+\frac{K}{3}\right) K^{2}$ & $+(2 K-1) N$ \\
\hline
\end{tabular}

More precisely, the complexity for the MMSE-FD-EXT is

- (per channel update) evaluating Eq. (13), except for the DFT operations: $2 M$ complex multiplications, $M$ complex additions

- to evaluate $\hat{\mathbf{x}}$ :

- FFT: $\frac{1}{2} M \log (M)$ complex multiplications, $\frac{1}{2} M \log (M)$ complex additions

- $M$ complex multiplications

- IFFT: $\frac{1}{2} M \log (M)$ complex multiplications, $\frac{1}{2} M \log (M)$ complex additions

In (the rather common) case only one spectral zero would be reconstructed, the MMSE-ZR would add to this:

- (per channel update) calculate $\mathbf{F}_{\mathrm{D}} \mathbf{F}_{\mathrm{D}}^{\mathcal{H}}$, needed for $\mathbf{q}: P$ complex multiplications, $P-1$ complex additions

- to evaluate $\hat{\mathbf{x}}$ :

- calculating q: $P$ complex multiplications, $P-1$ complex additions

- scaling and adding the complex exponential pertaining to the missing zero: $N$ complex multiplications, $N$ complex additions.

For $N=48$ and $P=16$, this means that the number of complex multiplications for a channel update, assuming $\Lambda_{\mathrm{f}}$ is known, increases from 128 to 144 ( $\left.+13 \%\right)$. The number of complex multiplications to evaluate $\hat{\mathbf{x}}$ it increases from 448 to $510(+14 \%)$. The figures for the additions are nearly identical.

\section{Simulations and discussion}

To illustrate the performance of the ZR technique, simulations have been done based on two channels found in literature as well as for the general case of Rayleigh fading. The section is concluded with a more general comparison of equalization techniques using ITU channels.

Channels $h_{1}(n)=[-0.3699+j 0.5782-0.4053+$ $j 0.5750-0.0834+j 0.04060 .1587+j 0.0156]$ and $h_{2}(n)$ $=[0.707000 .707]$ correspond to $p_{2}$ from [14] and channel A from [12], respectively. These channels are shown in Fig. 2. 


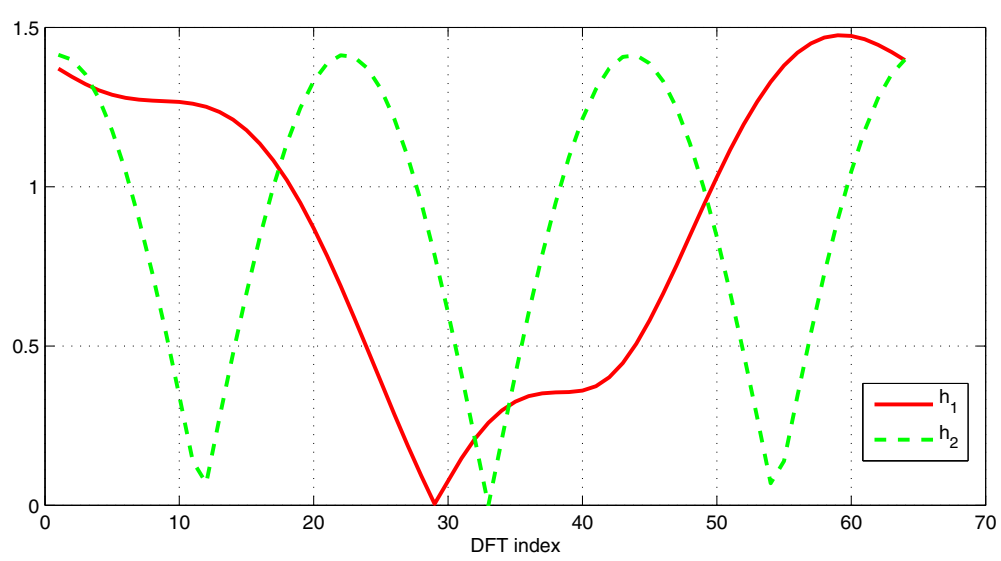

Fig. 2 Magnitude response for $h_{1}(n)$ (from [14]) and $h_{2}(n)$ (from [12])

The OFDM system in the original papers is replaced by ZP-SC with $M=64, P=L$ and $N=64-P$. Note that $h_{1}(n)$ has a spectral close-to-zero at the 30th subcarrier and $h_{2}(n)$ has a spectral zero as well as two spectral close-to-zeros. The noise is white Gaussian with different variances to match the SNR. The BER performance is simulated using a Monte Carlo method with 100,000 random blocks, assuming a 16-QAM system.

For the first channel, Fig. 3 indicates that limiting the noise at the spectral close-to-zero frequency, as the MMSE-FD-EXT does, is a good strategy for low SNR. However, because of the noise enhancement at the spectral close-to-zero, the MMSE-FD-EXT is unable to benefit fully from increasing SNR levels and at some point the ZFE-TD starts to perform better. The ZFEZR equalizer manages to stay very close to the ZFE-TD, which obviously provides a performance lower bound for any ZFE. As expected, the MMSE-ZR performs better than the ZFE-ZR, especially for low SNRs, and it even outperforms the ZFE-TD. Because the channel exhibits a spectral close-to-zero, and therefore the noise enhancement is finite, even the performance of the ZFE-FD-EXT eventually improves as the SNR increases, albeit slower.

The results for channel $h_{2}(n)$ are shown in Fig. 4. Because of the spectral zero, the noise enhancement for

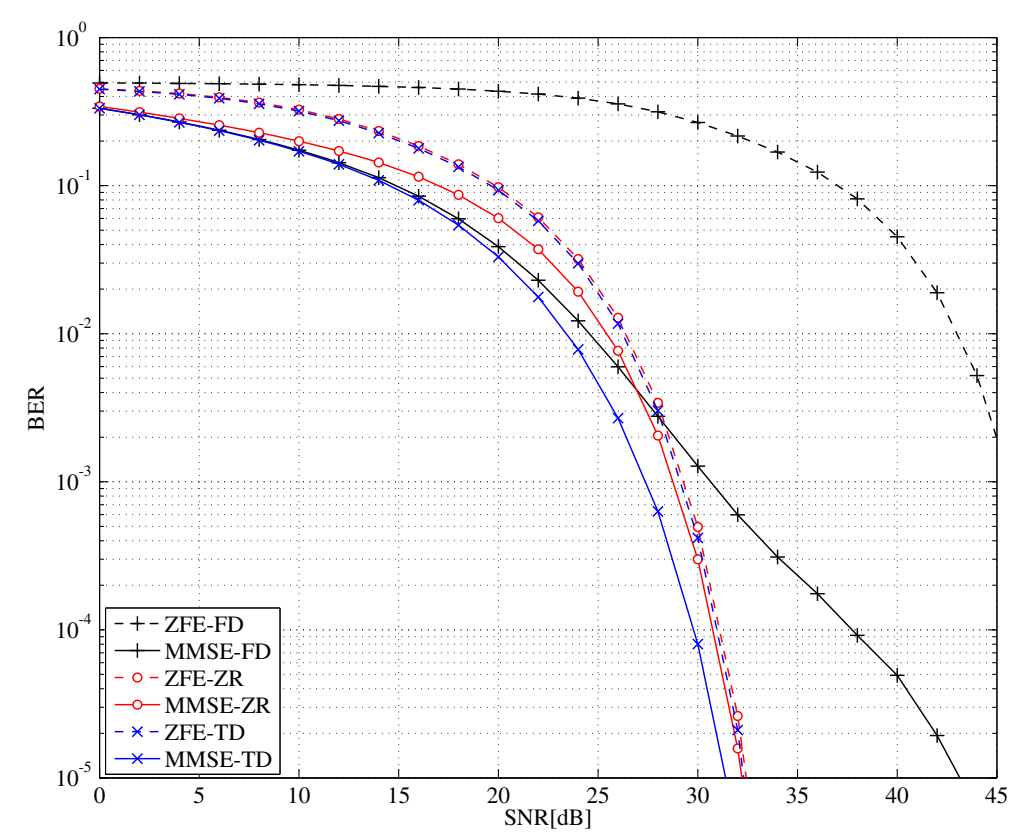

Fig. 3 Performance comparison for channel $h_{1}(n)$ 


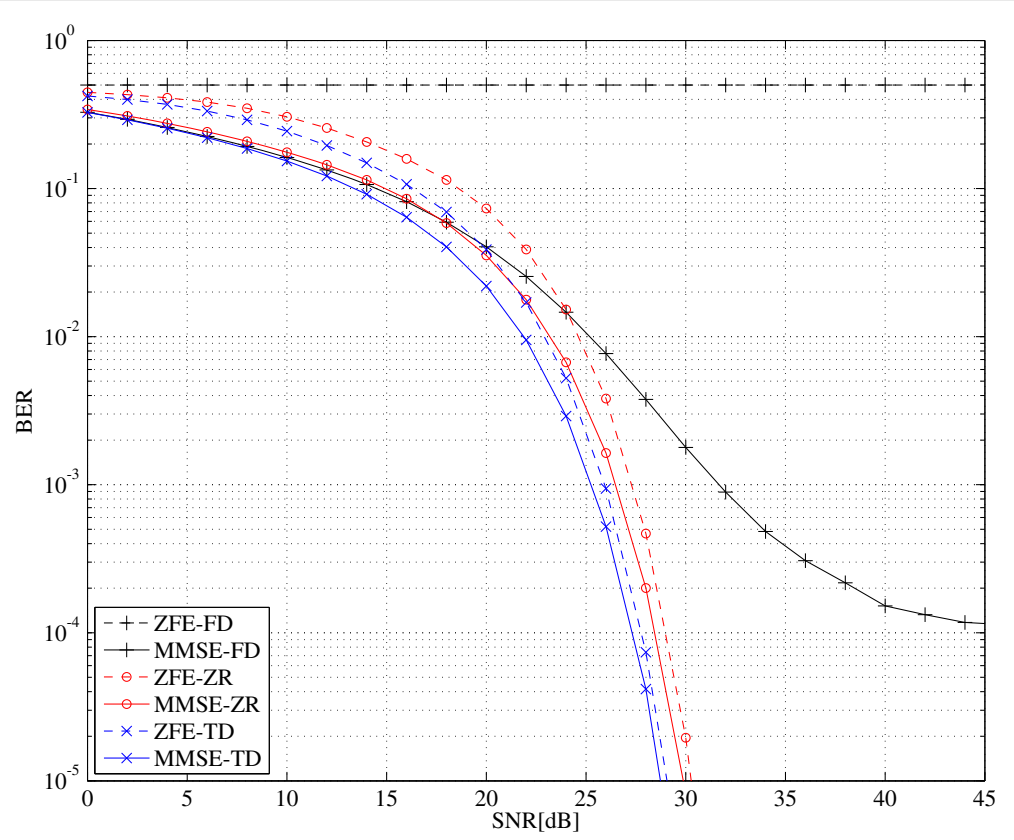

Fig. 4 Performance comparison for channel $h_{2}(n)$

the ZFE-FD-EXT equals infinity, as reflected by a BER of 0.5 for all SNR values. The MMSE-FD-EXT is also severely impacted by the spectral zero.

For moderate SNR values, its performance is comparable to that of Fig. 3, however, the performance now saturates for high SNR values. Indeed, all information is lost at the spectral zero and the best strategy is to discard the noise present at this sub-carrier. Roughly speaking, this implies that one $M$ th part of the signal power will be lost, resulting in an irreducible error, no matter how high the SNR. This is also apparent from the MSE curves in Fig. 5, for which this limit would be at $64^{-1}$ or $-18 \mathrm{~dB}$.
The ZFE-ZR and MMSE-ZR on the other hand assume three spectral (close-to-) zeros $(K=3)$ and reconstruct the information that was stored there. As the SNR levels increase, the estimation of the $\mathbf{q}$ vector in Eq. (18) also improves and therefore these equalizers do not show a saturation. They achieve a performance close to the ZFETD and the MMSE-TD, which are obviously not at all affected by the spectral zero. These curves also illustrate that the MSE for the two ZFEs is linearly dependent on the noise energy. For low SNR values, the ZFEs are clearly inferior to MMSE-based equalizers that limit the noise enhancement.

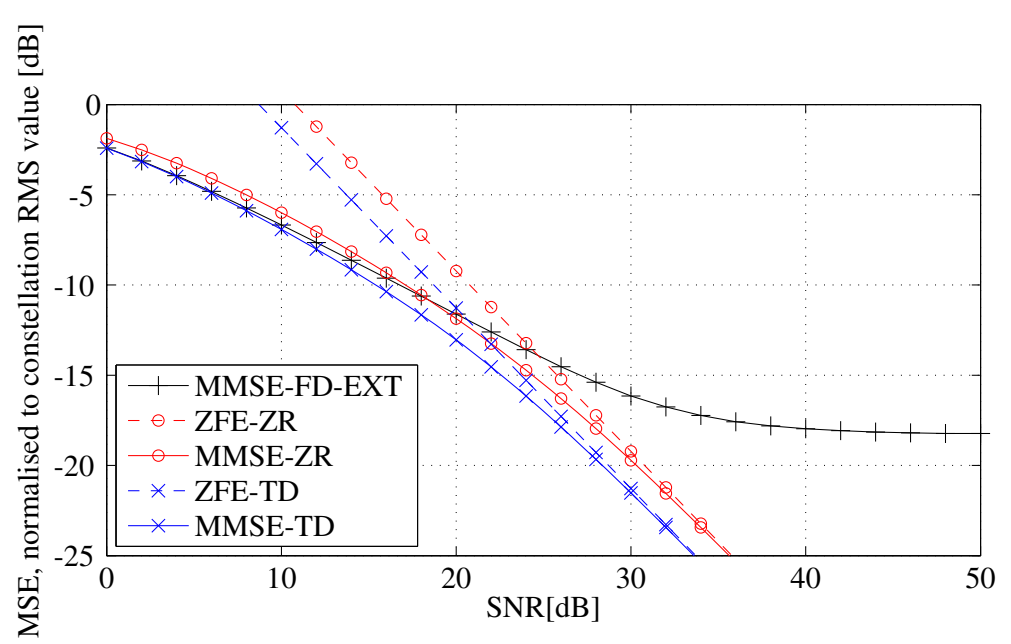

Fig. 5 Mean square error for channel $h_{2}(n)$ 
The Rayleigh fading model is a more realistic description of the wireless channel dominated by multipath ([23], p. 705). Such model has been simulated for 10,000 randomly generated channels of order $L=5$, with both the real and imaginary part of the impulse responses following a Gaussian distribution with variance $\sigma_{\mathrm{h}}^{2}=0.5$. For each channel, 1000 random blocks using 64-QAM coding have been simulated with $M=32, P=L=5, N=26$. The threshold to determine which elements of $\Lambda_{\mathrm{e}}$ are considered as zeros was set at 0.05 . The calculated BER is shown in Fig. 6. Again, the zero-restoration equalizers outperform the classical frequency-domain equalizers but are inferior to equalization in the time domain. Note that the zero-restoration mechanism is only triggered for a limited number of channels. The gain is therefore less impressive than for the previous results.

Finally, some more extensive simulations have been done, comparing ZP to $\mathrm{CP}, \mathrm{SC}$ to OFDM and matrix folding to matrix extension. The dimensions for IEEE 802.11a [24] have been used together with channel models presented by the ITU, more specifically the Indoor office test environment channel $B$ [25]. The useful signal length $N=$ 48 and the padding length $P=16$, be it a $\mathrm{ZP}$ or $\mathrm{CP}$. The constellation size for all symbols was QAM64. It is important to note that no coding was used and for OFDM no bit loading was done. The following modulation schemes are compared:

- SC-ZP, using MMSE-TD, ZFE-TD, MMSE-ZR, ZFE-ZR, MMSE-FD-EXT, ZFE-FD-EXT and MMSE-FD-FOLD
- SC-CP, using an MMSE-style FD equalizer

- OFDM-ZP, using MMSE-FD-EXT and MMSE-ZR.

- OFDM-CP, using an MMSE-style FD equalizer without the final IDFT step. This is the typical OFDM operation.

In total, 20,000 random channels were generated; for each one, 1000 symbols were transmitted. The results are presented in Fig. 7.

The best performance for SC-ZP systems is again obtained using the MMSE-TD and ZFE-TD, followed by the MMSE-ZR and ZFE-ZR and the other equalizers. It is interesting to see that the SC-ZP with MMSE-FDFOLD has the same performance as $\mathrm{SC}-\mathrm{CP}$, which makes sense because the operations on the useful signal are identical; only the noise distribution is different. The OFDM schemes systematically perform worse than the corresponding SC alternative. This result is pessimistic, though, because no bit loading is being used. Nevertheless, the OFDM-MMSE-ZR again performs better than the OFDM-MMSE-FD-EXT.

\section{Conclusions}

Two new frequency-domain equalizers have been proposed to equalize zero-padded OFDM and SC-block transmissions over a channel exhibiting spectral zeros or spectral close-to-zeros. Both exploit the redundancy of the $\mathrm{ZP}$ to restore the lost information. The first technique is shown to have the zero forcing property; the second one is very similar to an MMSE equalizer. A particularly

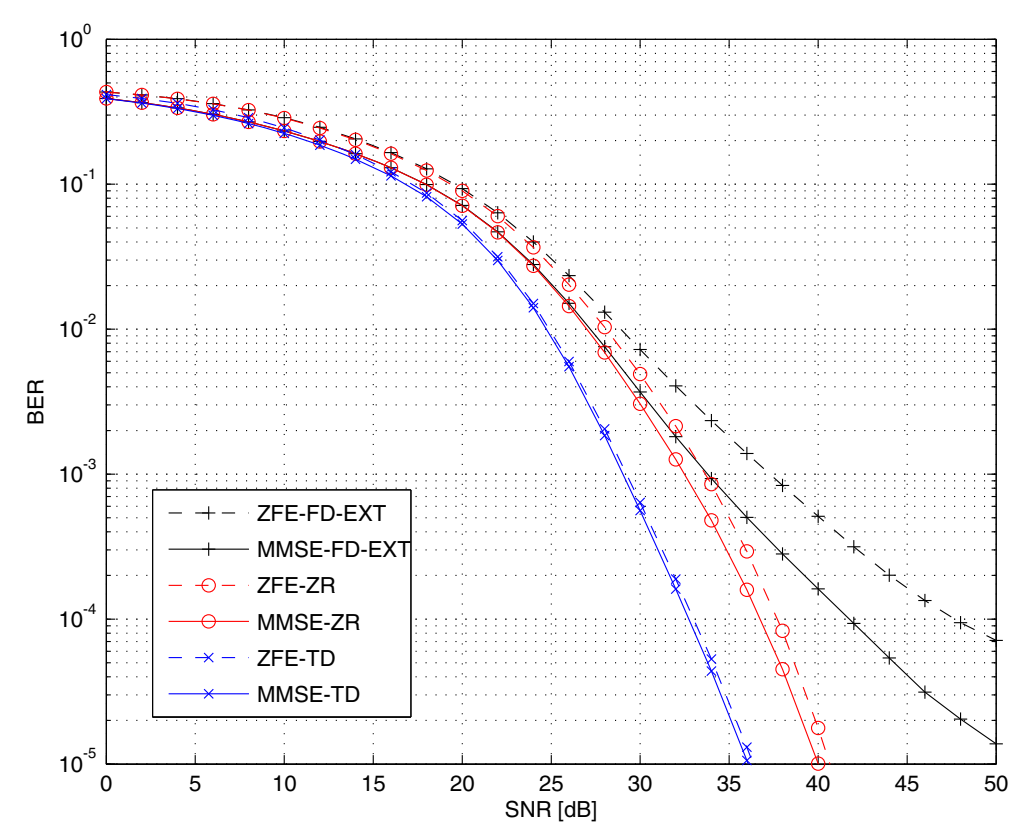

Fig. 6 Performance comparison for Rayleigh fading channels 


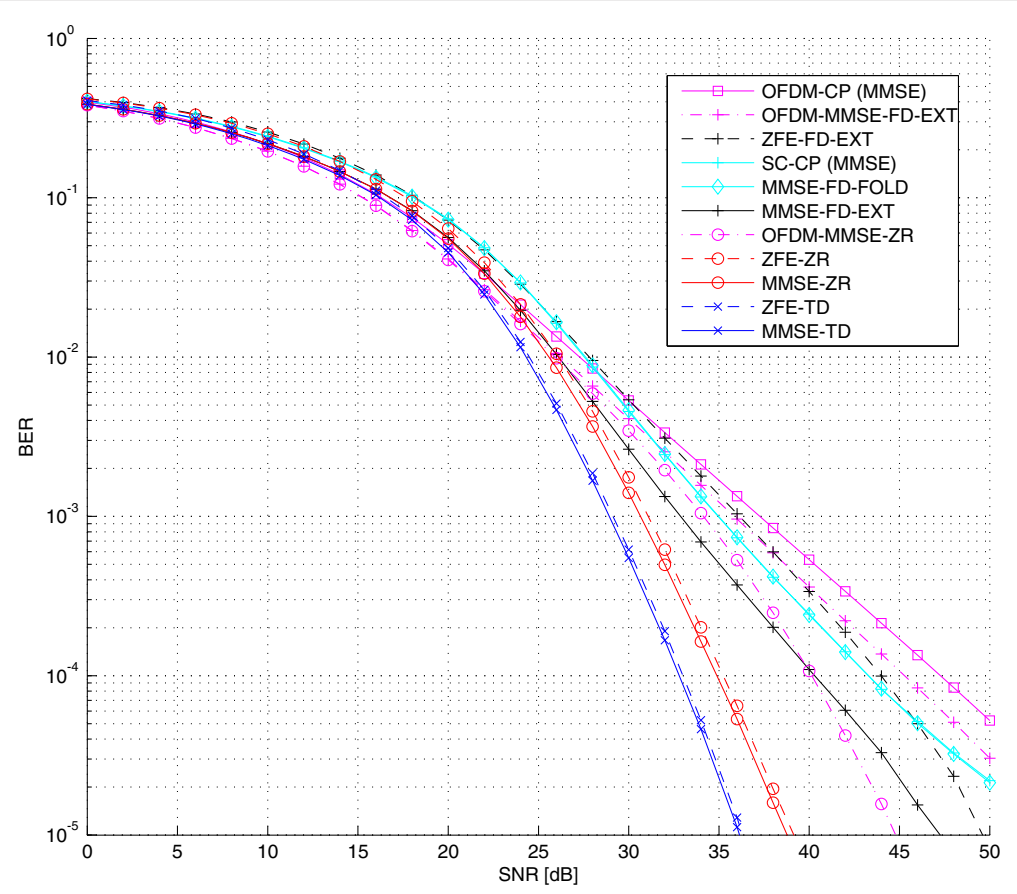

Fig. 7 Comparing several existing methods for IEEE802.11a dimensions and ITU channels

interesting feature is that these equalizers can be implemented by adding a post-processing to the output of a classical frequency-domain equalizer, typically requiring only very limited additional resources. Simulations show a performance comparable to time-domain equalizers, at a computational complexity comparable to the original frequency-domain equalizers. The equalizers can easily be modified to work with unique wording as well.

\section{Appendix}

Theoretical analysis of the performance

The bit error rate (BER) of a communications system can directly be calculated from the modulation scheme and the achieved mean squared error (MSE). For any equalizer $\mathbf{W}$, the MSE of the equalized blocks is given by the diagonal elements of the autocorrelation matrix of the equalization error, i.e.

$$
\begin{aligned}
\mathrm{MSE} & =\operatorname{Diag}\left\{E\left\{(\mathbf{W} \mathbf{y}-\mathbf{x})(\mathbf{W} \mathbf{y}-\mathbf{x})^{\mathcal{H}}\right\}\right\} \\
& =\operatorname{Diag}\left\{(\mathbf{W H}-\mathbf{I})(\mathbf{W} \mathbf{H}-\mathbf{I})^{\mathcal{H}}+\sigma_{\mathrm{n}}^{2} \mathbf{W} \mathbf{W}^{\mathcal{H}}\right\}
\end{aligned}
$$

The MSE has a contribution stemming from the inexact channel equalization and a noise contribution. The first contribution obviously equals zero for any ZFE.

\section{ZFE}

Bearing in mind that the ZFE-FD-EXT of Eq. (12) can be rewritten as

$$
\mathbf{W}_{\text {ZFE-FD-EXT }}=\left[\mathbf{F}_{\mathrm{A}}^{\mathcal{H}} \mathbf{F}_{\mathrm{C}}^{\mathcal{H}}\right]\left[\begin{array}{c|c}
\Lambda_{\mathrm{nZ}}^{-1} & \mathbf{O} \\
\hline \mathbf{O} & \Lambda_{\epsilon}^{-1}
\end{array}\right] \mathbf{F},
$$

its MSE equals

$$
\begin{aligned}
\underset{\text { ZFE-FD-EXT }}{\operatorname{MSE}}= & \operatorname{Diag}\left\{\sigma_{\mathrm{n}}^{2}\left[\mathbf{F}_{\mathrm{A}}^{\mathcal{H}} \mathbf{F}_{\mathrm{C}}^{\mathcal{H}}\right]\left[\begin{array}{c|c}
\Lambda_{\mathrm{nz}}^{-1} \mathbf{O} \\
\hline \mathbf{O} & \Lambda_{\epsilon}^{-1}
\end{array}\right]\right. \\
& {\left.\left[\begin{array}{c|c}
\Lambda_{\mathrm{nz}}^{-1} & \mathbf{O} \\
\hline \mathbf{O} & \Lambda_{\epsilon}^{-1}
\end{array}\right]^{\mathcal{H}}\left[\begin{array}{c}
\mathbf{F}_{\mathrm{A}} \\
\mathbf{F}_{\mathrm{C}}
\end{array}\right]\right\} } \\
= & \frac{\sigma_{\mathrm{n}}^{2}}{M}\left(\operatorname{Tr}\left\{\Lambda_{\mathrm{nz}}^{-1} \Lambda_{\mathrm{nz}}^{-1}{ }^{\mathcal{H}}+\Lambda_{\epsilon}^{-1} \Lambda_{\epsilon}^{-1} \mathcal{H}\right\}\right) 1_{\mathrm{N}} \times 1,
\end{aligned}
$$

with $\operatorname{Tr}\{$.$\} the trace operator and 1_{\mathrm{N}} \times 1$ an all-one vector. This MSE is identical for all elements in the received block. The MSE of the ZFE-ZR can be written as

$$
\begin{aligned}
\underset{\mathrm{ZFE}-\mathrm{ZR}}{\operatorname{MSE}} & \sigma_{\mathrm{n}} \operatorname{Diag}\left\{\mathbf{F}_{\mathrm{A}}^{\mathcal{H}} \boldsymbol{\Lambda}_{\mathrm{nz}}^{-1} \boldsymbol{\Lambda}_{\mathrm{nz}}^{-1 \mathcal{H}} \mathbf{F}_{\mathrm{A}}+\mathbf{F}_{\mathrm{A}}^{\mathcal{H}} \boldsymbol{\Lambda}_{\mathrm{nz}}^{-1} \boldsymbol{\Lambda}_{\mathrm{nz}}^{-1 \mathcal{H}} \mathbf{Q}^{\mathcal{H}} \mathbf{F}_{\mathrm{C}}\right. \\
& \left.\mathbf{F}_{\mathrm{C}}^{\mathcal{H}} \mathbf{Q} \boldsymbol{\Lambda}_{\mathrm{nz}}^{-1} \boldsymbol{\Lambda}_{\mathrm{nz}}^{-1} \mathbf{F}_{\mathrm{A}}+\mathbf{F}_{\mathrm{C}}^{\mathcal{H}} \mathbf{Q} \boldsymbol{\Lambda}_{\mathrm{nz}}^{-1} \boldsymbol{\Lambda}_{\mathrm{nz}}^{-1} \mathbf{Q}^{\mathcal{H}} \mathbf{F}_{\mathrm{C}}\right\}
\end{aligned}
$$

This MSE has a complex dependency on the noise of the sub-carriers corresponding to $\boldsymbol{\Lambda}_{\mathrm{nz}}$ and the correlation between the submatrices of $\mathbf{F}$. However, it is clear that it lacks the detrimental term in $\boldsymbol{\Lambda}_{\epsilon}^{-1}$. As the diagonal elements of $\boldsymbol{\Lambda}_{\epsilon}$ approach zero, the MSE of the ZFE-TDEXT increases unboundedly while the MSE of the ZFE-ZR is not influenced. Therefore, the latter has a superior performance in the presence of spectral (close-to-) zeros.

\section{MMSE}

The MSE of MMSE equalizers has a contribution stemming from the inexact channel equalization as well as 
a noise contribution. Both will now be analysed for the MMSE-FD-EXT and the MMSE-ZR.

\section{Signal contribution}

For the MMSE-FD-EXT, the signal contribution to the MSE equals

$$
\begin{aligned}
& \underset{\substack{\text { MMSE-FD-EXT } \\
\text { signal }}}{\operatorname{MSE}}=\operatorname{Diag}\left\{\left(\left[\begin{array}{ll}
\mathbf{F}_{\mathrm{A}}^{\mathcal{H}} & \mathbf{F}_{\mathrm{C}}^{\mathcal{H}}
\end{array}\right]\left[\begin{array}{c|c}
\Gamma_{\mathrm{nz}} \Lambda_{\mathrm{nz}} & \mathbf{O} \\
\hline \mathbf{O} & \Gamma_{\epsilon} \Lambda_{\epsilon}
\end{array}\right]\left[\begin{array}{c}
\mathbf{F}_{\mathrm{A}} \\
\hline \mathbf{F}_{\mathrm{C}}
\end{array}\right]-\mathbf{I}_{\mathrm{N}}\right)\right. \\
& \left.\times\left(\left[\begin{array}{cc}
\mathbf{F}_{\mathrm{A}}^{\mathcal{H}} & \mathbf{F}_{\mathrm{C}}^{\mathcal{H}}
\end{array}\right]\left[\begin{array}{c|c}
\boldsymbol{\Gamma}_{\mathrm{nz}} \boldsymbol{\Lambda}_{\mathrm{nz}} & \mathbf{O} \\
\hline \mathbf{O} & \boldsymbol{\Gamma}_{\epsilon} \boldsymbol{\Lambda}_{\epsilon}
\end{array}\right]^{\mathcal{H}}\left[\frac{\mathbf{F}_{\mathrm{A}}}{\mathbf{F}_{\mathrm{C}}}\right]-\mathbf{I}_{\mathrm{N}}\right)\right\} \\
& =\operatorname{Diag}\left\{\mathbf{F}_{\mathrm{A}}^{\mathcal{H}}\left(\Gamma_{\mathrm{nz}} \Lambda_{\mathrm{nz}}-\mathbf{I}_{\mathrm{V}}\right) \mathbf{F}_{\mathrm{A}} \mathbf{F}_{\mathrm{A}}^{\mathcal{H}}\left(\Gamma_{\mathrm{nz}} \Lambda_{\mathrm{nz}}-\mathbf{I}_{\mathrm{V}}\right)^{\mathcal{H}} \mathbf{F}_{\mathrm{A}}\right. \\
& +\mathbf{F}_{\mathrm{A}}^{\mathcal{H}}\left(\Gamma_{\mathrm{nz}} \Lambda_{\mathrm{nz}}-\mathbf{I}_{\mathrm{V}}\right) \mathbf{F}_{\mathrm{A}} \mathbf{F}_{\mathrm{C}}^{\mathcal{H}}\left(\Gamma_{\epsilon} \Lambda_{\epsilon}-\mathbf{I}_{\mathrm{K}}\right){ }^{\mathcal{H}} \mathbf{F}_{\mathrm{C}} \\
& +\mathbf{F}_{\mathrm{C}}^{\mathcal{H}}\left(\Gamma_{\epsilon} \Lambda_{\epsilon}-\mathbf{I}_{\mathrm{K}}\right) \mathbf{F}_{\mathrm{C}} \mathbf{F}_{\mathrm{A}}^{\mathcal{H}}\left(\Gamma_{\mathrm{nz}} \Lambda_{\mathrm{nz}}-\mathbf{I}_{\mathrm{V}}\right)^{\mathcal{H}} \mathbf{F}_{\mathrm{A}} \\
& \left.+\mathbf{F}_{\mathrm{C}}^{\mathcal{H}}\left(\Gamma_{\epsilon} \Lambda_{\epsilon}-\mathbf{I}_{\mathrm{K}}\right) \mathbf{F}_{\mathrm{C}} \mathbf{F}_{\mathrm{C}}^{\mathcal{H}}\left(\Gamma_{\epsilon} \Lambda_{\epsilon}-\mathbf{I}_{\mathrm{K}}\right)^{\mathcal{H}} \mathbf{F}_{\mathrm{C}}\right\} \\
& \underset{\sigma_{\mathrm{n}} \ll 1}{\approx \operatorname{Diag}}\left\{\mathbf{F}_{\mathrm{C}}^{\mathcal{H}} \mathbf{F}_{\mathrm{C}} \mathbf{F}_{\mathrm{C}}^{\mathcal{H}} \mathbf{F}_{\mathrm{C}}\right\} \text {, } \\
& \Lambda_{\epsilon} \approx \mathbf{O}_{\mathrm{K}}
\end{aligned}
$$

where the approximation is valid in case of spectral (closeto-) zeros and for high SNR values, i.e. small $\sigma_{\mathrm{n}}$, so that the following relations hold:

$$
\boldsymbol{\Gamma}_{\mathrm{nz}} \boldsymbol{\Lambda}_{\mathrm{nz}} \underset{\sigma_{\mathrm{n}} \ll 1}{\approx} \mathbf{I}_{\mathrm{V}}
$$

and

$$
\boldsymbol{\Gamma}_{\epsilon} \boldsymbol{\Lambda}_{\epsilon} \underset{\Lambda_{\epsilon} \approx \mathbf{O}_{\mathrm{K}}}{\approx} \mathbf{O}_{\mathrm{K}}
$$

From Eq. (32), it is seen that part of the information is irrevocably lost due to Eq. (34), leading to an irreducible error. If $K=1$ (only one spectral zero), this MSE contribution is identical for all elements of the block and equal to $M^{-1}$; otherwise, it is dependent on the correlation between the rows of $\mathbf{F}_{C}$.

For the MMSE-ZR, the signal contribution to the MSE can be written as

$$
\begin{aligned}
& \underset{\substack{\text { MMSE-ZR } \\
\text { signal }}}{\operatorname{MSE}}=\operatorname{Diag}\left\{\left(\left[\begin{array}{ll}
\mathbf{F}_{\mathrm{A}}^{\mathcal{H}} & \mathbf{F}_{\mathrm{C}}^{\mathcal{H}}
\end{array}\right]\left[\begin{array}{c|c}
\mathbf{I} & \mathbf{O} \\
\hline \mathbf{Q} & \mathbf{O}
\end{array}\right]\left[\begin{array}{c|c}
\Gamma_{\mathrm{nz}} \Lambda_{\mathrm{nz}} & \mathbf{O} \\
\hline \mathbf{O} & \Gamma_{\epsilon} \Lambda_{\epsilon}
\end{array}\right]\left[\begin{array}{c}
\mathbf{F}_{\mathrm{A}} \\
\hline \mathbf{F}_{\mathrm{C}}
\end{array}\right]-\mathbf{I}_{\mathrm{N}}\right)\right. \\
& \left.\times\left(\left[\begin{array}{cc}
\mathbf{F}_{\mathrm{A}}^{\mathcal{H}} & \mathbf{F}_{\mathrm{C}}^{\mathcal{H}}
\end{array}\right]\left[\begin{array}{c|c}
\Gamma_{\mathrm{nz}} \Lambda_{\mathrm{nz}} & \mathbf{O} \\
\hline \mathbf{O} & \Gamma_{\epsilon} \Lambda_{\epsilon}
\end{array}\right]^{\mathcal{H}}\left[\begin{array}{c|c}
\mathbf{I} & \mathbf{Q}^{\mathcal{H}} \\
\hline \mathbf{O} & \mathbf{O}
\end{array}\right]\left[\begin{array}{c}
\mathbf{F}_{\mathrm{A}} \\
\hline \mathbf{F}_{\mathrm{C}}
\end{array}\right]-\mathbf{I}_{\mathrm{N}}\right)\right\} \\
& =\operatorname{Diag}\left\{\left(\mathbf{F}_{\mathrm{A}}^{\mathcal{H}}\left(\Gamma_{\mathrm{nz}} \Lambda_{\mathrm{nz}}-\mathbf{I}\right) \mathbf{F}_{\mathrm{A}}+\mathbf{F}_{\mathrm{C}}^{\mathcal{H}} \mathbf{Q} \Gamma_{\mathrm{nz}} \Lambda_{\mathrm{nz}} \mathbf{F}_{\mathrm{A}}-\mathbf{F}_{\mathrm{C}}^{\mathcal{H}} \mathbf{F}_{\mathrm{C}}\right)\right. \\
& \left.\times\left(\mathbf{F}_{\mathrm{A}}^{\mathcal{H}}\left(\Lambda_{\mathrm{nz}}^{\mathcal{H}} \Gamma_{\mathrm{nz}}^{\mathcal{H}}-\mathbf{I}\right) \mathbf{F}_{\mathrm{A}}+\mathbf{F}_{\mathrm{A}}^{\mathcal{H}} \Lambda_{\mathrm{nz}}^{\mathcal{H}} \Gamma_{\mathrm{nz}}^{\mathcal{H}} \mathbf{Q}^{\mathcal{H}} \mathbf{F}_{\mathrm{C}}-\mathbf{F}_{-} C^{\mathcal{H}} \mathbf{F}_{\mathrm{C}}\right)\right\} \\
& \underset{\sigma_{\mathrm{n}} \ll 1}{\approx} \operatorname{Diag}\left\{(\mathbf{F}_{\mathrm{C}}^{\mathcal{H}} \underbrace{\mathbf{Q} \mathbf{F}_{\mathrm{A}}}_{\equiv \mathbf{F}_{\mathrm{C}}}-\mathbf{F}_{\mathrm{C}}^{\mathcal{H}} \mathbf{F}_{\mathrm{C}})\left(\mathbf{F}_{\mathrm{A}}^{\mathcal{H}} \mathbf{Q}^{\mathcal{H}} \mathbf{F}_{\mathrm{C}}-\mathbf{F}_{\mathrm{C}}^{\mathcal{H}} \mathbf{F}_{\mathrm{C}}\right)\right\} \\
& \underset{\sigma_{\mathrm{n}} \ll 1}{\approx} 0,
\end{aligned}
$$

in which the approximation is valid for high SNR values, i.e. small $\sigma_{\mathrm{n}}$.

Summarizing: in case the elements of $\Lambda_{\epsilon}$ are (closeto-) zero, the MSE due to inexact channel equalization decreases as the SNR increases. For the MMSE-FD-EXT, this MSE hits a lower bound for low noise levels. In the case of the MMSE-ZR, there is no such lower bound, implying that this equalizer has more benefit from a higher SNR. The reason for this difference is that the information stored at the corresponding sub-carriers is irrevocably lost for the MMSE-FD-EXT, while it can be recovered by the MMSE-ZR, and the quality of this recovered signal improves as the SNR increases.

\section{Noise contribution}

For the MMSE-FD-EXT, the noise contribution to the MSE equals

$$
\underset{\substack{\text { MMSE-FD-EXT } \\ \text { noise }}}{\operatorname{MSE}}=\frac{\sigma_{\mathrm{n}}^{2}}{M}\left(\operatorname{Tr}\left\{\Gamma_{\mathrm{nz}} \Gamma_{\mathrm{nz}}^{\mathcal{H}}\right\}+\operatorname{Tr}\left\{\Gamma_{\epsilon} \Gamma_{\epsilon}^{\mathcal{H}}\right\}\right) 1_{\mathrm{N}} \times 1,
$$

This MSE contribution is again identical for all elements in the transmitted block. Substituting Eqs. (24) and (25) and taking into account the definition of $\Lambda_{\mathrm{nz}}$ and $\Lambda_{\epsilon}$, Eq. (36) can be rewritten as a function of the spectral responses $\lambda_{\mathrm{e}_{\mathrm{i}}}$ :

$\underset{\substack{\text { MMSE-FD-EXT } \\ \text { noise }}}{\operatorname{MSE}}=\frac{1}{M}\left(\sum_{i=1}^{M} \sigma_{\mathrm{n}}^{2} \frac{\lambda_{\mathrm{e}_{\mathrm{i}}}^{H}}{\lambda_{\mathrm{e}_{\mathrm{i}}}^{\mathcal{H}} \lambda_{\mathrm{e}_{\mathrm{i}}}+\sigma_{\mathrm{n}}^{2}}\left(\frac{\lambda_{\mathrm{e}_{\mathrm{i}}}^{H}}{\lambda_{\mathrm{e}_{\mathrm{i}}}^{\mathcal{H}} \lambda_{\mathrm{e}_{\mathrm{i}}}+\sigma_{\mathrm{n}}^{2}}\right)^{\mathcal{H}}\right) 1_{\mathrm{N}} \times 1$.

Spectral zeros do not contribute to the MSE here. Spectral close-to-zeros do contribute, however, and dominate at high SNR values, i.e. for small values of $\sigma_{\mathrm{n}}$. This follows from the behaviour of the function $\sigma_{\mathrm{n}}^{2} \frac{\lambda_{\mathrm{e}_{\mathrm{i}}}^{H}}{\lambda_{\mathrm{e}_{\mathrm{i}}}^{\mathcal{H}} \lambda_{\mathrm{e}_{\mathrm{i}}}+\sigma_{\mathrm{n}}^{2}}\left(\frac{\lambda_{e_{\mathrm{i}}}^{H}}{\lambda_{e_{\mathrm{i}}}^{\mathcal{H}} \lambda_{\mathrm{e}_{\mathrm{i}}}+\sigma_{\mathrm{n}}^{2}}\right)^{\mathcal{H}}$, which is shown in Fig. 8. For large values of $\sigma_{\mathrm{n}}$, this function is small because the MMSE equalizer prevents noise enhancement. Obviously, it is also small for extremely small values of $\sigma_{\mathrm{n}}$. It reaches a maximum at an in between point, more specifically for $\sigma_{\mathrm{n}}=\left|\lambda_{\mathrm{e}_{\mathrm{i}}}\right|$.

For the MMSE-ZR, the noise contribution to the MSE equals

$$
\begin{aligned}
\underset{\substack{\text { MMSE-ZR } \\
\text { noise }}}{\operatorname{MSE}}= & \sigma_{\mathrm{n}} \operatorname{Diag}\left\{\mathbf{F}_{\mathrm{A}}^{\mathcal{H}} \Gamma_{\mathrm{nz}} \Gamma_{\mathrm{nz}}^{\mathcal{H}} \mathbf{F}_{\mathrm{A}}+\mathbf{F}_{\mathrm{A}}^{\mathcal{H}} \Gamma_{\mathrm{nz}} \Gamma_{\mathrm{nz}}^{\mathcal{H}} \mathbf{Q}^{\mathcal{H}} \mathbf{F}_{\mathrm{C}}\right. \\
& \left.\mathbf{F}_{\mathrm{C}}^{H} \mathbf{Q} \Gamma_{\mathrm{nz}} \Gamma_{\mathrm{nz}}^{\mathcal{H}} \mathbf{F}_{\mathrm{A}}+\mathbf{F}_{\mathrm{C}}^{\mathcal{H}} \mathbf{Q} \Gamma_{\mathrm{nz}} \Gamma_{\mathrm{nz}}^{\mathcal{H}} \mathbf{Q}^{\mathcal{H}} \mathbf{F}_{\mathrm{C}}\right\}
\end{aligned}
$$

It is not trivial to give a quantitative description of this contribution to the MSE. Obviously, in the case of a spectral zero, this MSE contribution will be larger than for the case of the MMSE-FD-EXT. However, with increasing SNR, i.e. decreasing values of $\sigma_{\mathrm{n}}$, this contribution 


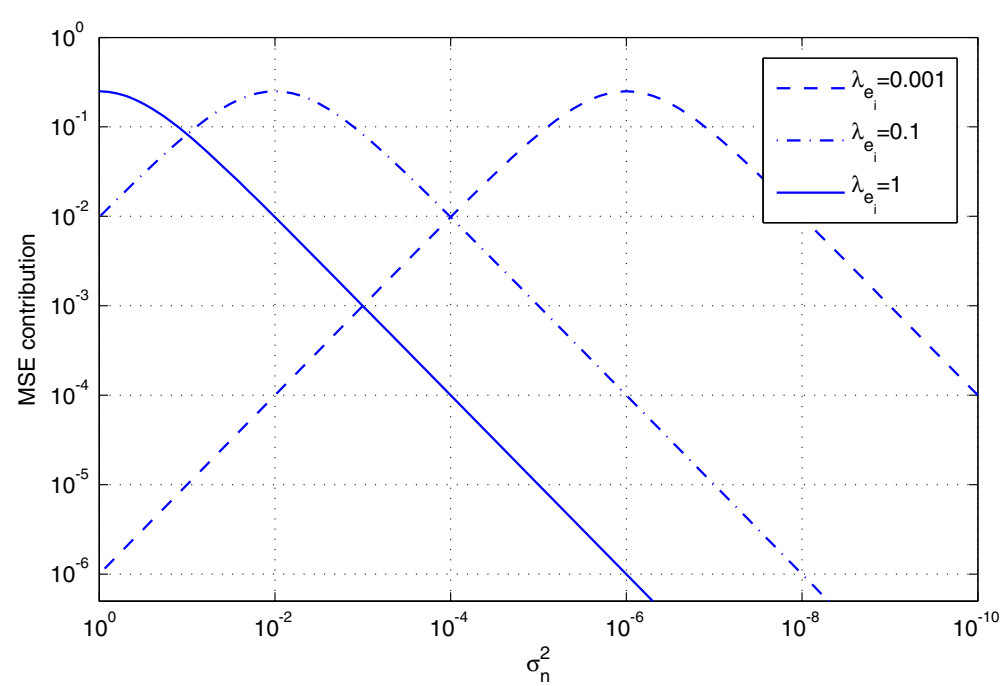

Fig. 8 The noise contribution to the MSE for different spectral responses $\lambda_{\mathrm{e}_{\mathrm{e}}}$. For high SNR (right hand side), the MSE is dominated by the noise contribution of the sub-carriers with the lowest spectral response (close-to-zeros)

will go down rapidly. For spectral close-to-zeros, the situation is even more favourable, especially at higher SNR values, because the dominant contributions from $\Lambda_{\epsilon}$, as described above, are not present here.

When looking at both the signal and the noise contributions to the MSE, it can be concluded that above a certain SNR threshold, the MMSE-ZR is expected to perform better than the MMSE-FD-EXT. Simulations have shown that for realistic scenarios this SNR threshold is typically somewhere between 10 and $30 \mathrm{~dB}$.
To illustrate this with an example, Fig. 9 shows the value of the MSE contributions, averaged over the block, for the MMSE-FD-EXT and the MMSE-ZR, for the channel ' $h$ ', used before. The presence of a close-to-zero starts dominating the MSE contribution of the noise for the MMSE-FD-EXT around SNR $=50 \mathrm{~dB}$. The contribution of the inexact channel equalization of the MMSEFD-EXT tends to saturate, but eventually $\sigma_{\mathrm{n}}$ becomes low enough such that the approximation of Eq. (34) no longer holds.

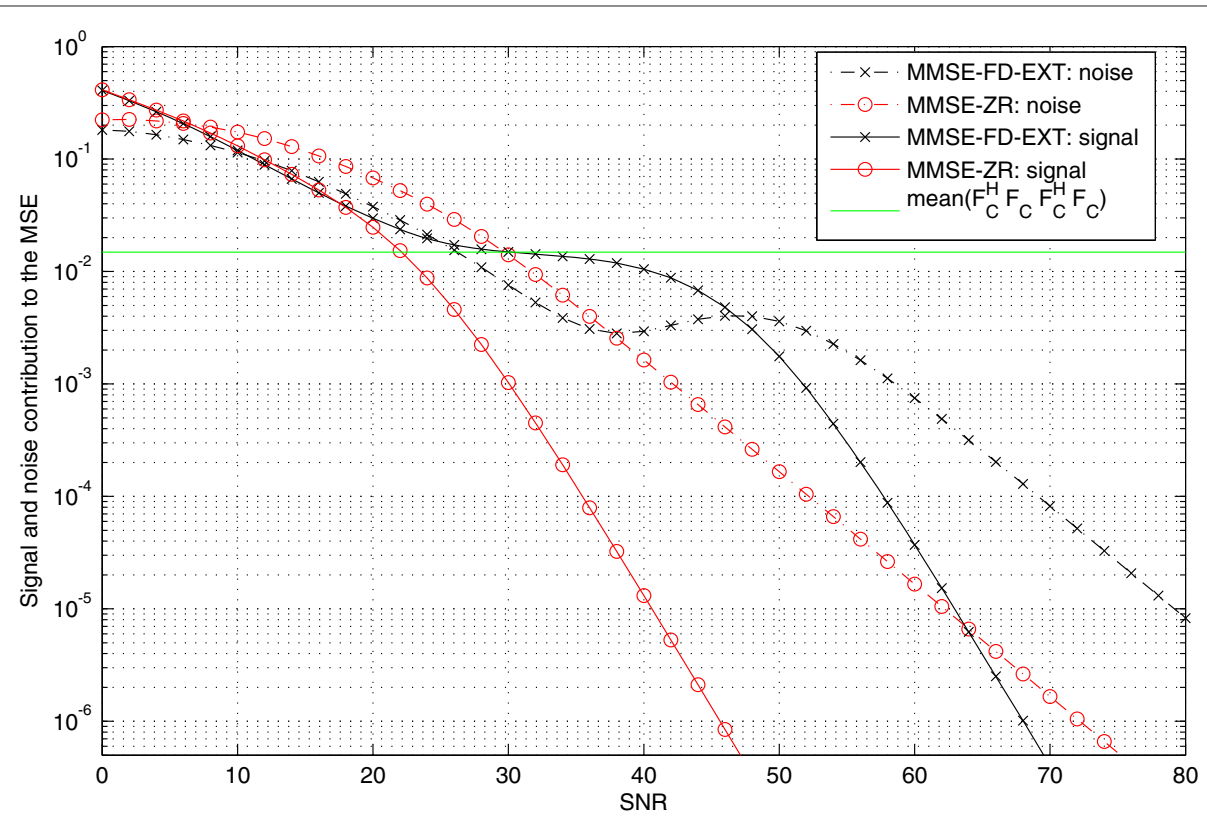

Fig. 9 Example of the MSE contributions from inexact channel equalization and noise enhancement for the MMSE-FD-EXT and the MMSE-ZR 


\section{Competing interests}

The authors declare that they have no competing interests.

\section{Authors' contributions}

The text was developed by the corresponding author, with reviewing and corrections by the second author. Both authors read and approved the final manuscript.

\section{Acknowledgements}

The authors are with the Department of Electrical Engineering (ESAT) and iMinds Future Health department, KU Leuven, Belgium. This research was carried out at ESAT, KU Leuven, in the frame of KU Leuven Research Council CoE EF/05/006 'Optimization in Engineering' (OPTEC) and PFV/10/002 (OPTEC), Concerted Research Action GOA-MaNet, the Belgian Programme on Interuniversity Attraction Poles initiated by the Belgian Federal Science Policy Office IUAP P7/19 'Dynamical systems, control and optimization' (DYSCO) 2012-2016 and IWT Project 'PHysical layer and Access Node TEchnology Revolutions: enabling the next generation broadband network' (PHANTER).

Received: 25 July 2015 Accepted: 27 March 2016

Published online: 14 April 2016

\section{References}

1. S Weinstein, P Ebert, Data transmission by frequency-division multiplexing using the discrete Fourier transform. Commun. Technol. IEEE Trans. 19(5), 628-634 (1971). doi:10.1109/TCOM.1971.1090705

2. A Peled, A Ruiz, in Acoustics, Speech, and Signal Processing, IEEE International Conference on ICASSP'80. Frequency domain data transmission using reduced computational complexity algorithms, vol. 5, (1980), pp. 964-967. doi:10.1109/ICASSP.1980.1171076

3. JAC Bingham, Multicarrier modulation for data transmission: an idea whose time has come. Commun. Mag. IEEE. 28(5), 5-14 (1990). doi:10.1109/35.54342

4. N LaSorte, WJ Barnes, HH Refai, in Global Telecommunications Conference, 2008. IEEE GLOBECOM 2008. IEEE. The history of orthogonal frequency division multiplexing, (2008), pp. 1-5. doi:10.1109/GLOCOM.2008.ECP.690

5. SB Weinstein, The history of orthogonal frequency-division multiplexing [history of communications]. Commun. Mag. IEEE. 47(11), 26-35 (2009). doi:10.1109/MCOM.2009.5307460

6. H Sari, G Karam, I Jeanclaude, Transmission techniques for digital terrestrial tv broadcasting. Commun. Mag. IEEE. 33(2), 100-109 (1995). doi:10.1109/35.350382

7. TWalzman, M Schwartz, Automatic equalization using the discrete frequency domain. Inform. Theory IEEE Trans. 19(1), 59-68 (1973). doi:10.1109/TIT.1973.1054952

8. D Falconer, SL Ariyavisitakul, A Benyamin-Seeyar, B Eidson, Frequency domain equalization for single-carrier broadband wireless systems. Commun. Mag. IEEE. 40(4), 58-66 (2002). doi:10.1109/35.995852

9. F Pancaldi, G Vitetta, R Kalbasi, N Al-Dhahir, M Uysal, H Mheidat, Single-carrier frequency domain equalization. Signal Process. Mag. IEEE. 25(5), 37-56 (2008). doi:10.1109/MSP.2008.926657

10. B Muquet, Z Wang, GB Giannakis, M de Courville, P Duhamel, Cyclic-prefixing or zero padding for wireless multicarrier transmissions? Commun. IEEE Trans. 50(12), 2136-2148 (2002). doi:10.1109/TCOMM.2002.806518

11. A Scaglione, GB Giannakis, S Barbarossa, Redundant filterbank precoders and equalizers. i. unification and optimal designs. Signal Process. IEEE Trans. 47(7), 1988-2006 (1999). doi:10.1109/78.771047

12. J Wang, J Song, Z-X Yang, L Yang, J Wang, Frames theoretic analysis of zero-padding ofdm over deep fading wireless channels. Broadcasting IEEE Trans. 52(2), 252-260 (2006). doi:10.1109/TBC.2006.872993

13. X-G Xia, Precoded and vector ofdm robust to channel spectral nulls and with reduced cyclic prefix length in single transmit antenna systems. Commun. IEEE Trans. 49(8), 1363-1374 (2001). doi:10.1109/26.939855

14. Y-P Lin, S-M Phoong, in Acoustics, Speech, and Signal Processing, 2003. Proceedings. (ICASSP'03). 2003 IEEE International Conference on. MMSE OFDM and prefixed single carrier systems: BER analysis, vol. 4, (Hong Kong, Hong Kong, 2003), pp. 229-324

15. GB Giannakis, Filterbanks for blind channel identification and equalization Signal Process. Lett. IEEE. 4(6), 184-187 (1997). doi:10.1109/97.586044
16. Z Wang, X Ma, GB Giannakis, in Wireless Communications and Networking Conference, 2002. WCNC2002. 2002 IEEE. Optimality of single-carrier zero-padded block transmissions, vol. 2, (2002), pp. 660-6642. doi:10.1109/WCNC.2002.993346

17. B Muquet, M de Courville, GB Giannakis, Z Wang, P Duhamel, in Acoustics, Speech, and Signal Processing, 2000. ICASSP '00. Proceedings. 2000 IEEE International Conference on. Reduced complexity equalizers for zero-padded OFDM transmissions, vol. 5, (2000), pp. 2973-29765. doi:10.1109/ICASSP.2000.861158

18. B Muquet, M de Courville, P Dunamel, G Giannakis, in Communications, 2000. ICC 2000. 2000 IEEE International Conference on. OFDM with trailing zeros versus OFDM with cyclic prefix: links, comparisons and application to the HiperLAN/2 system, vol. 2, (2000), pp. 1049-10532. doi:10.1109/ICC.2000.853658

19. C Tepedelenlioğlu, Q Ma, in Global Telecommunications Conference, 2005. GLOBECOM '05. IEEE. On the performance of linear equalizers for block transmission systems, vol. 6, (2005), pp. 5-3896. doi:10.1109/GLOCOM.2005.1578499

20. C Tepedelenlioğlu, in Acoustics, Speech, and Signal Processing, 2003. Proceedings. (ICASSP '03). 2003 IEEE International Conference on. Low complexity linear equalizers with maximum multipath diversity for zero-padded transmissions, vol. 4, (2003), pp. 636-94. doi:10.1109/ICASSP.2003.1202723

21. C Tepedelenlioğlu, Maximum multipath diversity with linear equalization in precoded OFDM systems. Inform. Theory, IEEE Trans. 50(1), 232-235 (2004). doi:10.1109/TIT.2003.821987

22. GH Golub, CFV Loan, Matrix computations, 3rd edn. (Johns Hopkins University Press, Baltimore, Maryland, USA, 1996)

23. JG Proakis, Digital communication, 2nd edn. (McGraw-Hill, McGraw-Hill of New York, New York, USA, 1989)

24. IEEE, IEEE Std 802.11a-1999(R2003) Part 11: Wireless LAN Medium Access Control (MAC) and Physical Layer (PHY) specifications, High-speed Physical Layer in the $5 \mathrm{GHz}$ band. The Institute of Electrical and Electronics Engineers, Inc. New York, NY 10016-5997, USA (2003)

25. International Telecommunications Union (ITU), Rec. ITU-R M.1225 Guidelines for evaluation of radio transmission technologies for IMT-2000. International Telecommunication Union (ITU) Geneva, Switzerland (1997)

\section{Submit your manuscript to a SpringerOpen ${ }^{\circ}$ journal and benefit from:}

- Convenient online submission

- Rigorous peer review

- Immediate publication on acceptance

- Open access: articles freely available online

- High visibility within the field

- Retaining the copyright to your article

Submit your next manuscript at $>$ springeropen.com 